\title{
О РЕШЕНИИ УРАВНЕНИЯ ПЕРЕНОСА В НЕОДНОРОДНОЙ СРЕДЕ МЕТОДОМ ДИСКРЕТНЫХ ОРДИНАТ
}

\author{
(Представил Г. Кузмин)
}

\section{Введение}

Метод дискретных ординат является одним из первых методов приближенного решения кинетического уравнения, он был развит для плоской геометрии $\left[{ }^{1,2}\right]$. В $\left[{ }^{3}\right]$ приведен алгоритм решения интегрального уравнеңия переноса на основе аппроксимации ядра интегрального уравнения квадратурными формулами, а в [4-6] исследована скорость сходимости при различных квадратурных формулах. Настоящая работа обобщает эти результаты на случай неоднородной среды, где коэффициенты (сечения) ослабления, поглощения и рассеяния являются кусочно-гладкими функциями.

\section{1. Некоторые особекности решаемой задачи}

Рассмотрим плоскопараллельную задачу, описывающую перенос излучения в неоднородной среде оптической толщины $H$ со сферической индикатрисой рассеяния [ $\left.{ }^{1}\right]$

$$
\begin{gathered}
\mu \frac{\partial \varphi(\tau, \mu)}{\partial \tau}+\sigma(\tau) \varphi(\tau, \mu)=(1 / 2) \sigma_{s}(\tau) \int_{-1}^{1} \varphi\left(\tau, \mu^{\prime}\right) d \mu^{\prime}+f_{1}(\tau), \\
\mu \in[-1,1], \quad \tau \in[0, H], \quad H=\text { const }<\infty, \\
\varphi(0, \mu)=0, \quad \mu>0 ; \quad \varphi(H, \mu)=0, \quad \mu<0 .
\end{gathered}
$$

Здесь $\sigma-$ коэффициент ослабления, а $\sigma_{s}-$ коэффициент рассеяния. Искомая функция $\varphi(\tau, \mu)$ - это интенсивность излучения на оптической глубине $\tau$ в направлении, образующем угол $\arccos \mu$ с осью, направленной перпендикулярно к слою среды в сторону возрастания $\tau$. Будем предполагать, что $0^{\prime}<\delta \leqslant \sigma_{s} \leqslant \sigma<\infty$. Пусть также функции $\sigma, \sigma_{s}$ и $f_{1}$ - кусочно-гладкие с возможными разрывами первого рода в точках $\tau_{i}, i=1,2, \ldots, M$, а на $\left[\tau_{i}, \tau_{i+1}\right], i=0,1, \ldots, M, \tau_{0}=0$, $\tau_{M+1}=H$ они имеют непрерывные производные.

Разделив обе части уравнения (1) на $\sigma$ и обозначив $x(\tau)=$ $=\sigma_{s}(\tau) / \sigma(\tau)$, получим с сохранением краевых условий (2) уравнение 


$$
\frac{\mu}{\sigma(\tau)} \frac{\partial \varphi(\tau, \mu)}{\partial \tau}+\varphi(\tau, \mu)=(\varkappa(\tau) / 2) \int_{-1}^{1} \varphi\left(\tau, \mu^{\prime}\right) d \mu^{\prime}+f(\tau),
$$

где $f(\tau)=f_{1}(\tau) / \sigma(\tau), \tau \in[0, H]$.

C помощью замены

$$
u(\tau)=(\varkappa(\tau) / 2) \int_{-1}^{1} \varphi\left(\tau, \mu^{\prime}\right) d \mu^{\prime}+f(\tau)
$$

краевая задача (2)-(3) сводится к уравнению

$$
u(\tau)=(x(\tau) / 2) \int_{0}^{H} \Sigma(\tau, s) u(s) d s+f(\tau), \quad \tau \in[0, H],
$$

где

$$
\Sigma(\tau, s)=\sigma(s) \int_{0}^{1}[\exp (-|g(\tau, s)| / \mu) / \mu] d \mu, \quad \tau, s \in[0, H]
$$

и

$$
g(\tau, s)=\int_{s}^{\tau} \sigma(\xi) d \xi
$$

причем при $\sigma(s) \equiv c=$ const имеет место равенство

$$
\Sigma(\tau, s)=c E(c|\tau-s|) \text {, }
$$

где $E(t)=\int_{0}^{1}[\exp (-t / \mu) / \mu] d \mu, t>0,-$ интегральная экспонента. Решив уравнение (4), решение краевой задачи (2)-(3) восстановим по формуле

$$
\varphi(\tau, \mu)=\left\{\begin{array}{cc}
\mu^{-1} \int_{0}^{\tau} \sigma(s) u(s) \exp (-g(\tau, s) / \mu) d s, & \mu>0, \\
u(\tau), & \mu=0, \\
-\mu^{-1} \int_{\tau}^{H} \sigma(s) u(s) \exp (g(s, \tau) / \mu) d s, & \mu<0 .
\end{array}\right.
$$

Рассмотрим теперь ядро (5) уравнения (4). Оно непрерывно по $\tau$ при $\tau \neq s$ и кусочно-гладко по $s$, причем имеет те же точки разрыва, что и функция $\sigma$. На участках непрерывности ядро имеет логарифмическую особенность

$\Sigma(\tau, s) \leqslant c \ln |\tau-s|, c=$ const; $\tau, s \in\left[\tau_{i}, \tau_{i+1}\right], i=1,2, \ldots, M$.

Далее, при любых $\tau$ и $s$ из $[0, H]$ функция

$$
\Sigma(\tau, s)>0
$$

и для любого $\tau \in[0, H]$

$$
\int_{0}^{\infty} \Sigma(\tau, s) d s<2
$$

Неравенство (10) вытекает из цепочки равенств

$$
\begin{aligned}
\int_{0}^{\infty} \Sigma(\tau, s) d s & =\int_{0}^{1} \mu^{-1}\left[\int_{0}^{\tau} \sigma(s) \exp (-g(\tau, s) / \mu) d s+\right. \\
& \left.+\int_{\tau}^{\infty} \sigma(s) \exp (-g(s, \tau) / \mu) d s\right] d \mu=
\end{aligned}
$$




$$
=2-\int_{0}^{1}[\exp (-g(\tau, 0) / \mu)-\exp (-g(\infty, \tau) / \mu)] d \mu .
$$

Условия (9) и (10) гарантируют однозначную разрешимость уравнения (4) в $L_{[0, H]}^{\infty}$ при любом достаточно гладком (в смысле $L^{\infty}$ ) свободном члене $f$, так как для интегрального оператора

$$
(T u)(\tau)=(\varkappa(\tau) / 2) \int_{0}^{H} \boldsymbol{\Sigma}(\tau, s) u(s) d s
$$

имеем

$$
\|T\|_{L^{\infty} \rightarrow L^{\infty}} \leqslant(1 / 2)\|\chi\|_{L^{\infty}} \operatorname{vraisup}\left|\int_{\tau \in[0, H]}^{H} \Sigma(\tau, s) d s\right|<\|\chi\|_{L^{\infty}} \leqslant 1 .
$$

Линейный оператор $T$ будет компактным как оператор из $L^{\infty} \rightarrow L^{\infty}$, поскольку его можно представить в виде

$$
(T u)(\tau)=(\varkappa(\tau) / 2) \sum_{i=0}^{M}\left(T_{i} u\right)(\tau),
$$

где $\left(T_{i} u\right)(\tau)=\int_{\tau_{i}}^{\tau_{i+1}} \boldsymbol{\Sigma}(\tau, s) u(s) d s, i=0,1, \ldots, M,-$ есть вполне непрерывный оператор из $C_{\left[\tau_{i}, \tau_{i+1}\right]} \rightarrow C_{[0, H]}$ как оператор со слабой особенностью.

Поскольку $f, \sigma, \sigma_{s} \in L_{[0, H]}^{\infty}$, то решение уравнения (4) $u$ также принадлежит $L_{[0, H]}^{\infty}$, причем из $\left[{ }^{7}\right]$ следует, что оно непрерывно дифференцируемо в промежутках $\left(\tau_{i}, \tau_{i+1}\right), i=0,1, \ldots, M, \tau_{0}=0, \tau_{M+1}=H$ и на этих промежутках удовлетворяет неравенствам

$$
\left|u^{\prime}(\tau)\right| \leqslant c_{1}+c_{2} \ln \left|\tau-\tau_{i}\right|, \tau \in\left(\tau_{i}, \tau_{i+1}\right), i=0,1, \ldots, M,
$$

где $c_{1}$ и $c_{2}-$ константы.

\section{2. Метод дискретных ординат}

Метод дискретных ординат решения краевой задачи (2)-(3) основывается на замене интеграла в (3) какой-либо квадратурной формулой $\left[{ }^{1,2}\right]$

$$
\int_{-1}^{1} \psi(\mu) d \mu \approx \sum_{j=1}^{n} \alpha_{j}\left[\psi\left(-\mu_{j}\right)+\psi\left(\mu_{j}\right)\right],
$$

где $\quad \alpha_{j}=\alpha_{j}^{(n)}>0, \quad \mu_{j}=\mu_{j}^{(n)} \in(0,1], \quad j=1,2, \ldots, n ; \quad n=1,2, \ldots$. Отсюда интегральное уравнение (4) заменяется на приближенное $u_{n}(\tau)=(\varkappa(\tau) / 2) \int_{0}^{H} \Sigma_{n}(\tau, s) u_{n}(s) d s+f(\tau), \tau \in[0, H], n=1,2, \ldots$,

где $\sum_{n}(\tau, s)=\sigma(s) \sum_{j=1}^{n}\left(\alpha_{j} / \mu_{j}\right) \exp \left(-|g(\tau, s)| / \mu_{j}\right) \quad$ - аппроксимация ядра (5) на основе квадратурной формулы 


$$
\int_{0}^{1} \psi(\mu) d \mu \approx \sum_{j=1}^{n} \alpha_{j} \psi\left(\mu_{j}\right) .
$$

По решению (12) решение приближенной к (2)-(3) краевой задачи восстанавливается аналогично (7).

Предположим, что квадратурная формула (13) точна для постоянной функции, т. е.

$$
\sum_{j=1}^{n} \alpha_{j}=1, \quad n=1,2, \ldots .
$$

Пусть $T_{n}-$ интегральный оператор уравнения (12)

$$
\left(T_{n} u\right)(\tau)=(\varkappa(\tau) / 2) \int_{0}^{H} \Sigma_{n}(\tau, s) u_{n}(s) d s .
$$

Условие (14) гарантирует однозначную разрешимость уравнения (12) в пространстве кусочно-гладких функций, ибо

$$
\left\|T_{n}\right\|_{L^{\infty} \rightarrow L^{\infty}}<1 .
$$

Опираясь на результаты $\left[{ }^{8,9}\right]$, при условии сходящегося квадратурного процесса (13), можно доказать сходимость

$$
\left\|T_{n}-T\right\|_{L^{\infty} \rightarrow L^{\infty}} \rightarrow 0, \quad n \rightarrow \infty .
$$

Из (15) следует, что оператор $I-T_{n}$ обратим и

$$
\left\|\left(I-T_{n}\right)^{-1}\right\|_{L^{\infty} \rightarrow L^{\infty}} \leqslant c=\text { const, }
$$

причем такое же неравенство имеет место для всех $L^{p}, p \in[1, \infty]$. Пусть, далее, $u$ и $u_{n}$ - решения интегральных уравнений (4) и (12) соответственно. Тогда

$$
u_{n}-u=\left(I-T_{n}\right)^{-1}\left(T_{n} u-T u\right),
$$

и скорость сходимости $u_{n} \rightarrow u$ зависит лишь от малости функции $T_{n} u-T u$.

\section{3. Решение уравнения (12)}

В этом разделе приведем алгоритм нахождения точного решения уравнения (12) при условии, что функции $\sigma$ и $\sigma_{s}-$ кусочно-постоянны. Пусть

$$
f(\tau)=a \exp (b \tau), \quad \tau \in[0, H],
$$

где $a$ и $b$ - кусочно-постоянные функции с разрывами в точках $\tau_{i}$, $i=1,2, \ldots, M$. Очевидно, что решение уравнения (12) кусочно-гладко. Будем искать его в виде

$$
\begin{gathered}
x_{i}^{n}(\tau)=c^{i} \exp \left(b^{i} \tau\right)+\sum_{r=1}^{n}\left[c_{r}^{i} \exp \left((\tau-H) d_{r}^{i}\right)+\right. \\
\left.+\bar{c}_{r}^{i} \exp \left(-\tau d_{r}^{i}\right)\right], \quad \tau \in\left[\tau_{i}, \tau_{i+1}\right], \quad i=0,1, \ldots, M, \\
\tau_{0}=0, \quad \tau_{M+1}=H,
\end{gathered}
$$


что является обобщением предложенного в $\left[{ }^{3}\right]$. Применим оператор $T_{n}$ к правой части равенства (18) и подставим полученное в уравнение (12). Приравняв коэффициенты при линейно-независимых экспоненциальных членах для каждого $i=0,1, \ldots, M$, получим условия для определения неизвестных коэффициентов в решении (18): $c_{r}^{i}, \bar{c}_{r}^{i}, d_{r}^{i}$, $r=1,2, \ldots, n, c^{i}, i=0,1, \ldots, M$. Из уравнения

$$
x_{i} \sum_{j=1}^{n} \frac{\alpha_{j}}{\left(d_{r}^{i} \mu_{j} / \sigma_{i}\right)^{2}-1}+1=0, \quad i=0,1, \ldots, M,
$$

где $x_{i}=x\left(\tau_{i}\right), \sigma_{i}=\sigma\left(\tau_{i}\right)$ и анализ которого проведен в $\left[{ }^{1,3}\right]$, найдем $n$ корней $d_{r}{ }^{i}>0, r=1,2, \ldots, n$. Из равенства

$$
c^{i}\left[1+x_{i} \sum_{j=1}^{n} \frac{\alpha_{j}}{\left(b^{i} \mu_{j} / \sigma_{i}\right)^{2}-1}\right]=a^{i}, \quad i=0,1, \ldots, M,
$$

находим $c^{i}, i=0,1, \ldots, M$. Подставим найденные значения $d_{r}{ }^{i}$ и $c^{i}$ в систему (19) порядка $2 n \times 2 n$ :

$$
\begin{aligned}
& \sum_{r=1}^{n}\left\{c_{r}^{i}\left[-B_{j}^{i}\left(d_{r}^{i}\right) E_{i}^{M+1}\left(d_{r}^{i}\right)+\sum_{k=1}^{i} B_{j}^{k-1}\left(d_{r}^{i}\right) F_{k, k}^{i, M+1}\left(d_{r}^{i}\right)\right]+\right. \\
& \left.+\bar{c}_{r}^{i}\left[\bar{B}_{j}^{i}\left(d_{r}^{i}\right) E_{i}^{0}\left(-d_{r}^{i}\right)-\sum_{k=1}^{i} \bar{B}_{j}^{k-1}\left(d_{r}^{i}\right) F_{k, k}^{i, 0}\left(-d_{r}^{i}\right)\right]\right\}- \\
& -c^{i}\left[B_{j}^{i}\left(b^{i}\right) E_{i}^{0}\left(b^{i}\right)-\sum_{k=1}^{i} B_{j}^{k-1}\left(b^{i}\right) F_{k, k}^{i, 0}\left(b^{i}\right)\right]=0, \\
& \sum_{r=1}^{n}\left\{c_{r}^{i}\left[\bar{B}_{j}^{i}\left(d_{r}^{i}\right) E_{i+1}^{M+1}\left(d_{r}^{i}\right)+\sum_{k=i+1}^{M} \bar{B}_{j}^{k}\left(d_{r}^{i}\right) \bar{F}_{i+1, k+1}^{k, M+1}\left(d_{r}^{i}\right)\right]-\right. \\
& \left.-\bar{c}_{r}^{i}\left[B_{j}^{i}\left(d_{r}^{i}\right) E_{i+1}^{0}\left(-d_{r}^{i}\right)-\sum_{k=i+1}^{M} B_{j}^{k}\left(d_{r}^{i}\right) \bar{F}_{i+1, k+1}^{h, 0}\left(-d_{r}^{i}\right)\right]\right\}+ \\
& +c^{i}\left[\bar{B}_{j}^{i}\left(b^{i}\right) E_{i+1}^{0}\left(b^{i}\right)+\sum_{k=i+1}^{M} \bar{B}_{j}^{k}\left(b^{i}\right) \bar{F}_{i+1, k+1}^{k, 0}\left(b^{i}\right)\right]=0,
\end{aligned}
$$

где

$$
B_{j}^{i}(y)=\frac{\sigma_{i}}{\sigma_{i} / \mu_{j}+y}, \quad \bar{B}_{j}^{i}(y)=\frac{\sigma_{i}}{-\sigma_{i} / \mu_{j}+y},
$$

$$
\begin{gathered}
F_{k, l}^{i, n}(y)=A_{k}^{i-1} E_{l}^{n}(y)-A_{k-1}^{i-1} E_{l-1}^{n}(y) ; \quad \bar{F}_{k, l}^{i, n}(y)=A_{k}^{i} E_{l}^{n}(y)-A_{k}^{i-1} E_{l-1}^{n}(y) ; \\
A_{k}^{i}=\exp \left[-\mu_{j}^{-1} \sum_{m=k}^{i} \sigma_{m}\left(\tau_{m+1}-\tau_{m}\right)\right], \\
E_{l}^{n}(y)=\exp \left[\left(\tau_{l}-\tau_{n}\right) y\right]
\end{gathered}
$$

и, решая ее для каждого $i=0,1, \ldots, M$, найдем все неизвестные коэффициенты решения (18). Система (19) однозначно разрешима при любом $i$, ибо если бы существовал такой номер $i=i^{*}$, при котором бы определитель системы равнялся нулю, то при свободном члене, равном тождественно нулю, система имела бы нетривиальное решение. Тогда однородное уравнение, соответствующее (12), имело бы нетривиальное решение 


$$
x(\tau)=\left\{\begin{array}{l}
0, \tau \in[0, H] \backslash\left[\tau_{i^{*}}, \tau_{i^{*}+1}\right], \\
x(\tau), \tau \in\left[\tau_{i^{*}}, \tau_{i^{*}+1}\right],
\end{array}\right.
$$

что противоречит неравенству (15).

\section{4. Исследование погрешности}

Преобразуем функцию $T_{n} u-T u$ следующим образом:

$$
\begin{aligned}
\left(T_{n} u-T u\right)(\tau)=(\varkappa(\tau) / 2) & \int_{0}^{H}[\Sigma n(\tau, s)-\Sigma(\tau, s)] u(s) d s= \\
= & (\varkappa(\tau) / 2)\left\{\sum_{j=1}^{m} \int_{\tau_{j-1}}^{\tau_{l}}[] u(s) d s+\int_{\tau_{m}}^{\tau}[] u(s) d s+\right. \\
& \left.+\int_{\tau}^{\tau_{m+1}}[] u(s) d s+\sum_{j=m+1}^{M} \int_{\tau_{f}}^{\tau_{l+1}}[] u(s) d s\right\},
\end{aligned}
$$

где $\tau_{j}, j=1,2, \ldots, M$, - точки разрыва функций $\sigma$ и $\sigma_{s}, \tau_{0}=0$, $\tau_{M+1}=H$. Число $m$ зависит от $\tau$ и определяет число разрывов до $\tau$. Далее, применяя к каждому интегралу интегрирование по частям, учитывая очевидное равенство

$$
\int_{a}^{b} \mu^{-1} \sigma(\tau) \exp [-g(\tau, c) / \mu] d \tau=\exp [-g(a, c) / \mu]-\exp [-g(b, c) / \mu]
$$

и равенство (14), получим

$$
\begin{gathered}
\left(T_{n} u-T u\right)(\tau)=\left\{u(0) v_{n}(\tau, 0)+u(H) v_{n}(H, \tau)+\right. \\
+\sum_{j=1}^{m}\left[v_{n}\left(\tau, \tau_{j}\right)\left(u\left(\tau_{j}+\right)-u\left(\tau_{j}-\right)\right)+\int_{\tau_{l-1}}^{\tau_{t}} v_{n}(\tau, \sigma) u^{\prime}(\sigma) d \sigma\right]- \\
-\sum_{j=m+1}^{M}\left[v_{n}\left(\tau_{j}, \tau\right)\left(u\left(\tau_{j}+\right)-u\left(\tau_{j}-\right)\right)+\int_{\tau_{l}}^{\tau_{l+1}} v_{n}(\sigma, \tau) u^{\prime}(\sigma) d \sigma\right]+ \\
\left.+\int_{\tau_{m}}^{\tau} v_{n}(\tau, \sigma) u^{\prime}(\sigma) d \sigma-\int_{\tau}^{\tau_{m+1}} v_{n}(\sigma, \tau) u^{\prime}(\sigma) d \sigma\right\}(\kappa(\tau) / 2),
\end{gathered}
$$

Рде

$$
v_{n}(a, b)=\int_{0}^{1} \exp (-g(a, b) / \mu) d \mu-\sum_{j=1}^{n} \alpha_{j} \exp \left(-g(a, b) / \mu_{j}\right) .
$$

Из $(20)-(21)$ видно, что точность приближения $u_{n}$ зависит от того, пасколько точно квадратурная формула (13) аппроксимирует интегралы от функции

$$
\Phi_{g}(\mu)=\exp (-g(\tau, a) / \mu),
$$

где функция $g(\tau, a)$, определенная в (6), - непрерывна. В случае

$$
g(\tau, a)=g(\tau-a) \equiv \tau-a
$$

в $\left[{ }^{5}\right]$ приводятся неулучшаемые оценки для аппроксимации $\Phi_{g}$ квадрагурной формулой средних прямоугольников, в $\left[{ }^{4}\right]-$ в случае квадратур Гаусса, а в $\left[{ }^{6}\right]$ доказана их неулучшаемость. 
Заметим, что $\Phi_{g} \in C_{[0,1]}^{\infty}, \Phi_{g}^{(k)}(0)=0$, однако производные возле точки $\mu=0$ не ограничены в совокупности,

$$
\left|\Phi_{g}^{(k)}(\mu)\right| \leqslant \gamma_{k} g^{-k} \quad(0 \leqslant \mu \leqslant 1,0<g \leqslant H, k=0,1, \ldots),
$$

где константы $\gamma_{k}$ зависят только от порядка производной, и из $[4,10]$ следует оценка

$$
\gamma_{k} \leqslant c k !, \quad c=\text { const. }
$$

Оценки полных вариаций для $\Phi_{g}$ и ее производных аналогичны $(22)$ в силу кусочной монотонности производных

$$
\begin{array}{ll}
\bigvee_{0}^{1}\left(\Phi_{g}\right) \leqslant 1, & 0 \leqslant g \leqslant H, \\
\bigvee^{1}\left(\Phi_{g}^{\prime \prime}\right) \leqslant \alpha g^{-2}, & 0<g \leqslant H .
\end{array}
$$

\section{5. Оценки скорости сходимости для некоторых квадратурных формул}

Нам понадобится нестандартная форма остаточного члена $r_{n}$ формулы средних прямоугольников на неравномерной сетке

$$
\int_{a}^{b} x(t) d t=\sum_{j=1}^{n}\left(t_{j}-t_{j-1}\right) x\left(\left(t_{j-1}+t_{j}\right) / 2\right)+r_{n},
$$

где

$$
a=t_{0}<t_{1}<\ldots<t_{n}=b .
$$

Имеет место неравенство [5]

$\left|r_{n}\right| \leqslant\left(h^{2} / 24\right) \int_{a}^{b}\left|x^{\prime \prime}(t)\right| d t+\left(h^{3} / 24\right) \bigvee_{a}^{b}\left(x^{\prime \prime}\right), \quad h=\max _{1 \leqslant j \leqslant n}\left(t_{j}-t_{j-1}\right)$.

Аналогично [5] справедлива

Л ем м а. Для определенной в (21) функции $v_{n}$ в случае квадратурной формулы (25) для любого $i=0,1, \ldots$, М справедливы оценки

$$
\begin{gathered}
\left\|v_{n}\left(\tau, \tau_{i}\right)\right\|_{c} \leqslant h, \\
\left|v_{n}\left(\tau, \tau_{i}\right)\right| \leqslant\left(c / \delta^{2}\right)\left(h^{2} \delta /\left|\tau-\tau_{i}\right|+h^{3} /\left|\tau-\tau_{i}\right|^{2}\right), \\
\left\|v_{n}\left(\tau, \tau_{i}\right)\right\|_{L} \leqslant\left(h^{2} / \delta\right)|\ln h|, \\
\left\|\int_{\tau_{t-1}}^{\tau_{t}}\left|v_{n}(\tau, \sigma)\right| u^{\prime}(\sigma) d \sigma\right\|_{c} \leqslant\left(c h^{2} / \delta\right)|\ln h|^{2}, \\
\left\|\int_{\tau_{t-1}}^{\tau_{t}}\left|v_{n}(\tau, \sigma)\right| u^{\prime}(\sigma) d \sigma\right\|_{L} \leqslant\left(c h^{2} / \delta\right)|\ln h|,
\end{gathered}
$$

где $\delta=\inf _{0 \leqslant \xi \leqslant H} \sigma(\xi)$, а $и$-решение уравнения (4). Постоянные во всех неравенствах имеют разные значения.

Доказ а тельство. Оценка (27) следует из (23), а оценка (28) из (24) и (26). Для $h<\tau_{i}<H-h$ в неравенстве 


$$
\begin{aligned}
\left\|v_{n}\left(\tau, \tau_{i}\right)\right\|_{L_{[0, H]}} & \leqslant\|\cdot\|_{L_{\left[0, \tau_{t}-h\right]}}+\|\cdot\|_{L_{\left[\tau_{t}-i, \tau_{t}+h\right]}}+ \\
& +\|\cdot\|_{L_{\left[\tau_{t}+h, H-h\right]}}+\|\cdot\|_{L_{[H-h, H]}}
\end{aligned}
$$

первый и третий члены оценим на основании (28) как $\left(c h^{2} / \delta\right)|\ln h|$, а второй и четвертый на основании (27) как $c h^{2}$. При $0 \leqslant \tau_{i} \leqslant h$ и $H-h \leqslant \tau_{i} \leqslant H$ в первом случае из неравенства

$$
\left\|v_{n}\left(\tau, \tau_{i}\right)\right\|_{L_{[0, H]}} \leqslant\|\cdot\|_{L_{[0,2 h]}}+\|\cdot\|_{L_{[2 h, H]}}
$$

и во втором случае из неравенства

$$
\left\|v_{n}\left(\tau, \tau_{i}\right)\right\|_{L[0, H]} \leqslant\|\cdot\|_{L_{[0, H-2 h]}}+\|\cdot\|_{L_{[H-2 h, H]}},
$$

варьируя оценками (27) и (28), приходим к оценке (29). Рассматривая отдельно случаи $\tau_{i-1}<\tau_{i} \leqslant 2 h$ и $2 h<\tau_{i} \leqslant H$ и применяя неравенства (11) и (27)-(28), придем к (30). Далее, меняя порядок интегрирования и используя оценки (11) и (29), придем к оценке (31). Перенос этого доказательства на случай $\tau_{i}>\tau, \quad i=m+1, \ldots, M$, для $v_{n}\left(\tau_{i}, \tau\right)$ не представляет трудности.

Лемма доказана.

Т еорем а 1. Для решений интегральных уравнений (4) $u$ (12) в случае квадратур средних прямоугольников (25) справедливы оценки

$$
\begin{gathered}
\left|u_{n}(\tau)-u(\tau)\right| \leqslant\left(c \theta / \delta^{2}\right) \sum_{i=0}^{M} \min \left\{\delta^{2} h, \delta h^{2} /\left|\tau_{i}-\tau\right|+\right. \\
\left.+h^{3} /\left(\tau_{i}-\tau\right)^{2}+\delta h^{2}(\ln h)^{2}\right\},
\end{gathered}
$$

2วe

$$
\begin{aligned}
& \theta=\max \left\{u(0), u(H),\left|u\left(\tau_{i}+\right)-u\left(\tau_{i}-\right)\right|, \quad i=1,2, \ldots, M\right\}, \\
& \delta=\inf _{0 \leqslant \xi \leqslant H} \sigma(\xi)>0, \quad c=\text { const, } \quad h=\max _{1 \leqslant j \leqslant n}\left(t_{j}-t_{j-1}\right), \quad t_{0}=0
\end{aligned}
$$

$u$

$$
\begin{gathered}
\left\|u_{n}-u\right\|_{L^{\infty}} \leqslant c(M+1) h, \\
\left\|u_{n}-u\right\|_{L} \leqslant(c / \delta)(M+1) h^{2}|\ln h|,
\end{gathered}
$$

и для решений краевой задачи (1)-(2) и ее приближения - оценки

$$
\begin{gathered}
\left|\varphi_{n}(\tau, \mu)-\varphi(\tau, \mu)\right| \leqslant \\
\leqslant\left(c \theta / \delta^{2}\right) \sum_{i=0}^{M} \min \left\{\delta^{2} h,\left(h^{2} \delta|\ln | \tau_{i}-\tau \|+h^{3} /\left|\tau-\tau_{i}\right|\right) /|\mu|\right\}, \\
\max _{-1 \leqslant \mu \leqslant 1}\left\|\varphi_{n}-\varphi\right\|_{L} \leqslant(c / \delta)(M+1) h^{2}|\ln h| .
\end{gathered}
$$

Дока за тел в с тво. Равномерные и интегральные оценки (33)-(34) немедленно следуют из оценки (16), равенства (17), вида погрешности (20)-(21) и неравенств $(27)-(31)$. Для получения поточечной оценки (32) равенство (17) перепишем в виде

$$
u_{n}-u=\left(T_{n}-T\right) u+\left(I-T_{n}\right)^{-1} T_{n}\left(T_{n} u-T u\right) .
$$

Отсюда 


$$
\begin{gathered}
\left|u_{n}(\tau)-u(\tau)\right| \leqslant\left|\left(T_{n} u-T u\right)(\tau)\right|+ \\
+\left\|\left(I-T_{n}\right)^{-1}\right\|_{L^{\infty} \rightarrow L^{\infty}}\left\|T_{n}\left(T_{n} u-T u\right)\right\|_{L^{\infty}}, \quad 0 \leqslant \tau \leqslant H .
\end{gathered}
$$

Пусть $T_{n} u-T u=W_{n}$, тогда

$$
\begin{gathered}
\left\|T_{n} W_{n}\right\|_{L^{\infty}} \leqslant(1 / 2)\|x\|_{L^{\infty}} \sup _{0 \leqslant \tau \leqslant H}\left|\int_{0}^{H} \Sigma_{n}(\tau, s) W_{n}(s) d s\right| \leqslant \\
\leqslant c \sup _{0 \leqslant \tau \leqslant H}\left\{\left|\int_{[0, H] \backslash\{-h, \tau+h]} \sum_{n}(\tau, s) W_{n}(s) d s\right|+\right. \\
\left.+\left|\int_{[\tau-h, \tau+h] \cap[0, H]} \sum_{n}(\tau, s) W_{n}(s) d s\right|\right\} \leqslant c\left\|W_{n}\right\|_{L}|\ln h|+c\left\|W_{n}\right\|_{L^{\infty}} h|\ln h|,
\end{gathered}
$$

так как для $\sum_{n}$ верно неравенство, аналогичное (8). Для $W_{n}$ справедливы оценки (33) и (34), отсюда

$$
\left\|T_{n} W_{n}\right\|_{L^{\infty}} \leqslant(c / \delta)(M+1) h^{2}(\ln h)^{2} .
$$

Теперь на основании $(20)-(21)$ и $(27)-(31)$ придем к оценке (32). Из (6) и оценок (32)-(34) следуют оценки скорости сходимости (35) и (36) .

Теорема 1 доказана.

Далее, следуя $\left[{ }^{4,6}\right]$, можно сформулировать и доказать соответствующую теорему о быстроте сходимости в случае квадратур Гаусса на $[0,1]$.

Теорем а 2. Для решений интегральных уравнений (4) $u$ (12) $u$ краевой задачи (1)-(2) вместе с аппроксимирующей ее задачей в случае квадратурной формулы Гаусса на $[0,1]$ имеют место оценки

$$
\begin{gathered}
\left\|u_{n}-u\right\|_{L^{\infty}} \leqslant c(M+1) h^{2}|\ln h|, \\
\max _{-1 \leqslant \mu \leqslant 1}\left\|\varphi_{n}-\varphi\right\|_{L_{[0, H]}^{\infty}} \leqslant c(M+1) h^{2}|\ln h| .
\end{gathered}
$$

Разобьем теперь отрезок $[0,1]$ на $l+1$ отрезков точками $\xi_{j}, j=$ $=1,2, \ldots, l+1$,

$$
0<\xi_{1}<\xi_{2}<\ldots<\xi_{l}<\xi_{l+1}=1
$$

таким образом, чтобы

$$
\xi_{j+1}=\xi_{j}^{2 n_{1} / 2 n_{1}+1}+\xi_{j}, \quad j=1,2, \ldots, l ; \quad n_{1}=1,2, \ldots .
$$

На каждом отрезке $\left[\xi_{j}, \xi_{j+1}\right], j=1,2, \ldots, l$, применим $n_{1}$-точечную квадратурную формулу Гаусса с постоянным весом на этом отрезке. Тогда, соответствующим образом выбрав है। и следуя $\left[{ }^{10}\right]$, можно утверждать, что имеет место

Т еорем а 3. Пусть квадратурная формула (13) построена согласно приведенному выше алгоритму. Тогда для решений интегральных уравнений (4) и (12) имеет место оценка

$$
\left\|u_{n}-u\right\|_{L^{\infty}} \leqslant c(M+1) 2^{-2 \sqrt{n}} n^{1 / 8}, \quad c=\text { const, }
$$

где $M$ - число точек разрыва сечений, $n=n_{1} \times l-$ число узлов (весов) квадратурной формулы (13), $n_{1}$ - число узлов (весов) на каждом отрезке, a $l$ - число отрезков. 


\section{Заключение}

Надо отметить, что при решении уравнения переноса со сферической индикатрисой рассеяния в плоскопараллельном однородном слое методом дискретных ординат оценки скорости сходимости приближенного решения к точному переносятся на класс задач с кусочно-гладкими функциями сечений, имеющими возможные разрывы первого рода в конечном числе точек. При таком расширении класса решений (c $C_{[0, H]}$ на $\left.L_{[0, H]}^{\infty}\right)$ лишь несколько видоизменяется поточечная оценка, а равномерные (в смысле $L^{\infty}$ ) и интегральные оценки ухудшаются в $M$ раз, где $M$ - число точек разрыва функций сечений. При этом выбор квадратурных формул для достижения более быстрой скорости сходимости зависит не от количества и расположения точек разрыва, а лишь от степени аппроксимации интегральной экспоненты. В этом заключается преимущество метода дискретных ординат для решения интегрального уравнения переноса в неоднородной среде перед методом механических квадратур, в котором выбор узлов сильно зависит от точек разрыва.

Автор выражает глубокую благодарность Г. Вайникко за постоянное внимание к работе.

\section{Л И Т Е Р А Т Р А}

1. Л ебедев В. И., М а р чук Г. И., Численные методы в теории переноса нейтронов, М., Атомиздат, 1971.

2. Ч а нд р а с ек а р С., Перенос лучистой энергии, М., ИЛ, 1953.

3. В а й н икко Г., К а рл енко Л., Ш и лм ан А., Изв. АН ЭССР, Физ. Матем., 25, № 2, 118-123 (1976).

4. Ч у я н в В. А., В кн.: Некоторые математические задачи нейтронной физики, М., Изд-во МГУ, 1960 , с. $199-220$.

5. В а йникко Г. М., М а рш а к А. Л., Изв. ВУЗов, Математика, № 11, 11-22 (1978).

6. М а рш а к А. Л., Уч. зап. Тартуск. ун-та, 500, 92-104 (1979).

7. М а р ч к Г. И., Аго шков В. И., Докл. АН СССР, 232, № 6, 1253-1256 (1977).

8. Влладим и ров В. С., Математические задачи односкоростной теории переноса частиц, М., Изд. МИ АН СССР, 1961.

9. Anselone, P. M., Collectively Compact Operator Approximation Theory, PrenticeHall, New Jersey, 1971.

10. Маршак А. Л., В кн.: Тезисы конференции «Теоретические и прикладные вопросы математики», Тарту, 1980, с. 199-201.

Институт астрофизики и физики атмосферь Академии наук Эстонской ССР

Поступила в редакцию $4 /$ III 1981

\section{A. MARSAK}

\section{KIIRGUSULEKANDE VORRANDI LAHENDAMINE MITTEHOMOGEENSES KESKKONNAS DISKREETSETE ORDINAATIDE MEETODIL}

Artiklis on üldistatud $\left.{ }^{3,5,6}\right]$ toodud tulemused juhule, kui hajumis- ja neeldumiskoefitsiendid on tükiti siledad funktsioonid. On esitatud kiirgusülekande vôrrandi lahendamise algoritm diskreetsete ordinaatide meetodil ning hinnatud erinevate kvadratuurvalemite koonduvuskiirust. 


\section{A. MARSAK}

\section{ON THE SOLUTION OF THE RADIATION TRANSFER EQUATION BY THE METHOD OF DISCRETE ORDINATES IN A NON-HOMOGENEOUS MEDIUM}

In the present paper the results obtainèd in $\left.{ }^{3,5,6}\right]$ are generalized to the case of a non-homogeneous medium where coefficients of extinction, absorption and scattering are piecewise smooth functions. An algorithm is presented for the solution of transfer equation (4) by the method of discrete ordinates consisting in the substitution of the kernel (5) of the integral operator by a quadrature rule (13) and in the exact solution of the approximate equation (12). It has been proved that the convergence speed of the approximate solution to the exact solution depends only on the accuracy with which the quadrature rule (13) approximates the integral of the function

$$
\Phi_{g}(\mu)=\exp (-g(\tau, a) / \mu), \quad \tau \in[0, \mathrm{H}], \quad \mu \in[0,1], \quad a=\text { const } \geqslant 0,
$$

where

$$
g(\tau, a)=\int_{a}^{\tau} \sigma(\xi) d \xi
$$

Here $\sigma$ is the coefficient of extinction.

In the case of the rectangular formula on a non-uniform net (25), uniform (33), and integral (34) estimates of convergence speeds are derived. Uniform estimates are also given on the application of the Gaussian quadrature formula normalized to the interval $[0,1]$ as well as to the specially selected intervals. 